\title{
Stochastic Geometry based Medium Access Games
}

\author{
M.K. Hanawal ${ }^{\dagger *}$, E. Altman ${ }^{\dagger}$ and F. Baccelli ${ }^{\ddagger}$ \\ $\dagger$ INRIA B.P.93, 2004 Route des Lucioles 06902 Sophia-Antipolis Cedex, FRANCE \\ * LIA, University of Avignon, 339, chemin des Meinajaries, Avignon, FRANCE \\ $\ddagger$ ENS-INRIA 45 rue dUlm, 75230 Paris, FRANCE
}

\begin{abstract}
This paper studies the performance of Mobile Ad hoc Network (MANET) when the nodes, that form a Poisson point process, selfishly choose their Medium Access Probability (MAP). We introduce a pricing scheme based on the transmission energy requirements and compute the symmetric Nash equilibrium MAP in closed form. It is shown that by appropriately pricing the nodes the selfish behavior of the nodes can be used to achieve the social optimum at equilibrium. Goodput and delay are considered as the performance metric that each node is interested in optimizing taking into account the cost it incurs. The Price of anarchy is then analyzed for these games. When utility with delay is considered, we bound the price of anarchy and study the effect of system parameters. When utility with goodput is considered it is shown that PoA is infinite at the price that achieves global optimal density of success.
\end{abstract}

Index Terms-Game Theory; Mobile Ad hoc Networks (MANET); pricing; Poisson Point Process; Stochastic Geometry

\section{INTRODUCTION}

In this paper we study competition over network resources at the medium access control (MAC) layer. It is well known that computing the Nash equilibria in games is in general a hard problem. Indeed, this problem falls into a class of problems introduced by Christos Papadimitriou in 1994, called PPAD (Polynomial Parity Arguments on Directed graphs). In view of this complexity, it becomes attractive to identify classes of games for which one may compute the equilibria at a low complexity. We thus study a MAC game under some statistical assumptions on the mobility pattern, which are on one hand reasonable in many real scenarios, and on the other hand, allow for tractable and in several cases, even explicit expressions for the Nash equilibrium.

We consider slotted time, and assume that the mobiles are synchronized. The basic assumptions on our model are

- The location of the transmitters at each time slot forms a homogeneous Poisson point process,

- Mobility is high, so that the location of the mobiles at different slots is an independent and identically distributed (IID) process.

- Medium access is controlled using Aloha

- Transmission success is based on SINR being larger than some threshold.

In particular, our assumptions characterize a uniform distribution of mobiles over the space: given their number in any finite region, their locations are IID and uniform.

We study several game problems that arise in ad-hoc networks, in which mobiles are located on the plane according to a spatial Poisson point process. We assume saturated sources, i.e. every mobile has always a packet to send.

We first consider the problem in which each mobile is a player: it chooses the transmission probability so as to maximize its own performance metric. We consider utility functions that model the tradeoff between quality of service indicators (such as the expected goodput or the expected delay) and power consumption related to the transmission.

Our goals are to obtain a symmetric Nash equilibrium (SNE) and study its properties. We are interested in particular in the case where the power consumption disutility represents a pricing decision of the network that wishes to determine a pricing that will induce an efficient equilibrium (in terms of the achieved goodput). Alternatively, the pricing may be taken such as to maximize the network's revenues.

Our main findings are:

1) Considering the goodput as as the quality of service to be maximized, we observe the tragedy of the commons [13]: the utility at equilibrium is zero for large values of the pricing parameter. Thus the price of anarchy is infinite. We show however that there exists a pricing parameter for which the goodput at equilibrium equals the one obtained under global cooperative throughput maximization.

2) Considering the expected delay as the quality of service to be minimized, we observe that the price of anarchy is bounded. Here too, there exists a pricing that allows one to obtain the global optimal expected delay at equilibrium. We show that $\mathrm{SNE}$ is not unique. The range of price parameters for which two SNE exist is characterized.

As the price of anarchy is infinity for certain price factors it may seem therefore that high prices have a negative effect on the network performance. However if we consider instead the network performance or the monetary profit of the operator, we discover that pricing can induce an equilibria for which these measures coincide with the global optimal values.

The Poisson assumption on the location of nodes allows us to obtain utilities in a surprisingly simple explicit form, which in turn allows us to obtain much insight on the property of the equilibria and on the role of the pricing. In [17], in the context of cellular networks explicit expression are obtained for coverage and throughput with the Poisson assumption on the base stations. We discuss the validity of the Poisson assumption in the concluding Section. 
There are several papers that model the nodes in the Aloha system as selfish users. In [16] a game theoretic model is proposed to analyze the performance of the Aloha network. It is assumed that when collusion occurs some of the transmissions can be successful. Authors in [15] study the performance of slotted Aloha with $n$ nodes that are selfish. Any collision results in the loss of the packet and there is a cost associated with the unsuccessful transmission. They characterize the equilibrium point as a function of $n$ and study its liming behavior. In [18] slotted Aloha is considered with $n$ selfish users and the distributed choice of the retransmission probabilities are analyzed. By adding a retransmission cost it is shown, numerically, that the throughput at equilibrium equals the optimal team throughput. Pricing is used in the context of the power control games in [20] to improve the performance at equilibrium.

All these paper do not take into account the geometric aspects of the node location, which is important when the nodes are mobile. We consider in this paper the geometric aspects of the MANET and analyze the network performance at equilibrium. For games that take geometric aspects into consideration see [19].

The paper is organized as follow. In Section II Poisson bipolar MANET is set up and performance metrics of interest are discussed. Section III considers the team case in which all nodes use the same MAP. Section IV considers a scenario in which all nodes are selfish. A medium access game is modeled between the nodes. Section V studies this game with a utility function that is based on the goodput and transmission energy costs. Section VI studies the medium access game with another utility that includes delay as performance metric and transmission costs. Section VII analyzes the price of anarchy for these games. We end with some remarks on validity of Poisson process assumptions and future work in Section VIII.

\section{MODEL AND SETUP}

Consider the simplified mobile ad hoc network (MANET) model called the Poisson bipolar model proposed in [2]. Assume that each node follows the slotted version of the Aloha medium access control (MAC) protocol. Each dipole of the MANET consists of a transmitter and an associated receiver. We assume that each node has an infinite backlog of packets to transmit to its receiver. Nodes are scattered in the Euclidian space according to an homogeneous Poisson point process of intensity $\lambda$. Each node is associated with a multi dimensional mark that carries information about the transmission status, fading condition, and the distance to receiver. We follow the notation of [12]. Let $\tilde{\Phi}=\left\{X_{i}, M_{i}\right\}_{i \geq 1}$ denote an independently marked Poisson point, where

- $\Phi=\left\{X_{i}\right\}_{i \geq 1}$ denotes the Poisson point process of intensity $\lambda$, representing the location of nodes in the Euclidean plane.

- $\left\{M_{i}=\left(S_{i}, e_{i}\right)\right\}_{i \geq 1}$ denotes the independent marks of the Poisson point process $\Phi$, which are made of three components:
- $S=\left\{S_{i}\right\}_{i \geq 1}$ denotes the channel condition between nodes and their associated receivers. It is assumed that channel conditions are independently and identically distributed (IID) across the nodes with a generic distribution denoted as $F$ with mean $1 / \mu$.

- $e=\left\{e_{i}\right\}_{i \geq 1}$ are indicator functions that take value 1 if a given node decides to transmit in a given time slot; otherwise they take value zero. They are assumed to be IID across the nodes.

- The processes $S, e$ are assumed to be independent of each other.

- We assume that distance between the transmitter and its associated receiver is at a fixed distance $r$.

Let $l(x, y)$ denote the attenuation function between any two given points $x, y \in \mathbb{R}^{2}$. We assume that this function just depends on the distance between points, i.e., $|x-y|$. With a slight abuse of notation we denote this function as $l(x, y)=$ $l(|x-y|)$. We assume the following form for this attenuation function

$$
l(x, y)=(A|x-y|)^{-\beta} \text { for } A>0 \text { and } \beta>2 .
$$

The marks $\left\{e_{i}\right\}_{i \geq 1}$ indicate if a given node transmits in a given time slot. Then the probability that the $i$ th node transmits is $p:=\operatorname{Pr}\left\{e_{i}=1\right\}=\mathbb{E}[e]$ (Medium Access Probability (MAP)). Note that $\Phi$ defines a pair of independent Poisson process representing transmitters $\Phi^{1}=\left\{X_{i}, e_{i}=1\right\}$ and non transmitters $\Phi^{0}=\left\{X_{i}, e_{i}=0\right\}$ with intensities $p \lambda$ and $(1-p) \lambda$ respectively. We assume that the channel between the receiver of a given node and the transmitter of any other node is also distributed as random variable $S$. All the nodes transmit at a fixed power denoted as $P$. Signal transmitted by a transmitter located at $X_{i}$ is successfully received at its associated receiver at location $y_{i}$ if the signal to interference and noise ratio (SINR) at location $y_{i}$ is larger than some threshold $T$, i.e.,

$$
S I N R_{i}:=\frac{P S l\left(\left|X_{i}-y_{i}\right|\right)}{I_{\tilde{\Phi}^{1}}+W}>T,
$$

where $W$ denotes the thermal noise power at the receiver and $I_{\tilde{\Phi}^{1}}$ denotes the shot noise of the Poisson point process $\Phi^{1}$ namely $I_{\tilde{\Phi}^{1}}=\sum_{X_{i} \in \Phi^{1}} P S l\left(\left|X_{i}-y_{i}\right|\right)$.

Consider a typical node at the origin, $X_{0}=0$ with mark $M_{0}=\left(S_{0}, e_{0}\right)$. The typical node is said to be covered if (2) holds given that the it is a transmitter. Then the coverage probability of the typical node is

$$
\mathbf{P}^{0}\left\{\frac{P S l(r)}{I_{\tilde{\Phi}^{1}}+W}>T \mid e_{0}=1\right\},
$$

where $\mathbf{P}^{0}$ denotes the Palm distribution [8][Chap. I] of the stationary marked Poisson point process $\tilde{\Phi}$. By using Slivnyak's theorem [8], the coverage probability of a typical nodes when all other nodes use MAP $p$ is evaluated in [2][12] and denoted as $p_{c}(r, p \lambda, T)$. Let the MAP of the typical node be $p^{\prime}$. Then the typical node is a transmitter with probability $p^{\prime}$ and a non transmitter with probability $\left(1-p^{\prime}\right)$. We call the product of 
the MAP and the coverage probability of the typical node the goodput and denote it as

$$
g\left(p^{\prime}, p\right):=p^{\prime} p_{c}(r, p \lambda, T) .
$$

For the typical node we shall also consider the delay in successfully transmitting the packets to its associated receiver. We assume that if a packet transmission fails then the packet is retransmitted till success. We also assume that nodes can re sample the channel in each slot. Recall our assumption on the mobility model that locations of the nodes are IID across the time slot. Then by Little's theorem [14] the mean delay for a typical node is given by reciprocal of its goodput. Let $t\left(p^{\prime}, p\right)$ denote the delay of the typical node that uses MAP $p^{\prime}$ when all other nodes use the MAP $p$. Then

$$
t\left(p^{\prime}, p\right):=1 / g\left(p^{\prime}, p\right) .
$$

In the following sections we consider two scenarios. First we assume that the nodes of the MANET cooperate, i.e., use the same MAP that is assigned to them in each time slot. The value of a MAP that optimizes the spatial network performance are evaluated. We then consider a game scenario in which each node is selfish and aims to choose a MAP that optimize their own performance taking into account the transmission costs. We study the effect of the transmission costs on the network performance at equilibrium and look for a price value that results in improved spatial network performance at equilibrium.

\section{Rate Control: The team CASE}

In this section we assume that all the nodes belong to a single operator, and transmit at the MAP set by the operator. If nodes have information about other nodes in their vicinity, they can achieve a better goodput by cooperating: Nodes moving from dense cluster to sparse clusters can increase their MAP as chances of collision reduce. Nodes moving from sparse clusters to dense clusters can reduce their MAP as chances of collision increase. We assume that, because of mobility, nodes can't gather such information in each time slot and transmit with a MAP that is set by the operator. With some abuse of notation we denote the goodput again by $g(p)$, which is the same for all nodes as all of them transmit with the same MAP. In the rest of the paper when we write goodput with a single argument the team case is assumed. When we write it with two arguments, as in (4), game scenario is assumed (see section IV). We use the same convention when delay is considered as the performance metric. The following Lemma immediately follows from [2][Lemma 3.2]

Proposition 1: Let each node in MANET Poisson bipolar model transmit with MAP $p$ and $S$ be Rayleigh distributed with mean $1 / \mu$; then the goodput is

$$
\begin{aligned}
& g(p)= \exp \left\{-2 \pi \lambda p \int_{0}^{\infty} \frac{u}{1+l(r) /(T l(u))} \mathrm{d} u\right\} \\
& \times \psi_{W}(\mu T / P l(r)),
\end{aligned}
$$

where $\psi_{W}(\cdot)$ denotes the Laplace transform of the noise power $W$.
Corollary 1: For exponential S, zero noise power $W \equiv 0$, and the path loss model in Equation (1), the goodput of a typical node is

$$
g(p)=p \exp \left\{-2 \pi \lambda p r^{2} T^{2 / \beta} K(\beta)\right\},
$$

where $K(\beta)=\frac{\Gamma(2 \beta) \Gamma(1-2 / \beta)}{\beta}$ and $\Gamma(x)=\int_{0}^{\infty} z^{x-1} e^{-z}$. Hence forth we adopt the assumptions of Corollary 1 in all the subsequent calculations. However, our results hold when $W$ has any distribution with finite mean, as it appears as a constant multiplicative factor in Equation (7). For notational convenience we write $C:=C(\beta, T, r)=2 \pi r^{2} T^{2 / \beta} K(\beta)$.

The operator is interested in optimizing the social performance of the network. In particular, we assume that the operator aims at maximizing the mean goodput per unit area or minimizing the mean delay per unit area. The performance seen by a typical node can be used to derive the spatial performance of the Poisson bipolar MANET. Campbell's formula [8][Sec. 2.1.2] for stationary Poisson point processes ensures that the performance experienced by a typical node is also that of the average performance of the Poisson MANET. The mean goodput per unit area is then the product of the intensity of the Poisson point process and the goodput, i.e., $\lambda g(p)$. This quantity is referred to as the density of success and denoted by $d_{\text {suc }}(r, p \lambda, T)$ in [9][Chap. 16]. We denote this term simply as $d_{s}(p)$. Similarly, the mean delay per unit surface area is given by $\lambda t(p)$. We denote this spatial performance metric as $d_{t}(p)$ and refer to it as spatial delay density. Note the the density of delay success is the reciprocal of density of success multiplied by a factor $\lambda^{2}$. Hence the MAP that maximizes the $d_{s}(p)$ also minimizes $d_{t}(p)$. The MAP that optimizes the density of success is given in [9][Prop 16.8] and [9][Corol. 16.9] :

Proposition 2: Under the assumption of Corollary 1 the MAP that maximizes the density of success and minimizes the density of delay is given by

$$
p_{m}=\min \{1,1 / \lambda C\},
$$

and the corresponding optimal density of success is given by

$$
d_{s}\left(p_{m}\right)= \begin{cases}1 /(e \lambda C), & \text { if } \lambda C>1 \\ \lambda \exp \{-\lambda C\}, & \text { if } \lambda C \leq 1,\end{cases}
$$

and the corresponding optimal delay density is given by

$$
d_{t}\left(p_{m}\right)= \begin{cases}\lambda^{2} e C, & \text { if } \quad \lambda C>1 \\ \lambda \exp \{\lambda C\}, & \text { if } \quad \lambda C \leq 1\end{cases}
$$

\section{Rate Control: The Non-Cooperative Case}

In this section we assume that each node of the Poisson MANET is a selfish player. We use node and player interchangeably. The decision variable of each player is its MAP $p$. Choosing the transmission probability is a form of rate control.

Let $U\left(p^{\prime}, p\right)$ be the utility for a typical node that uses the MAP $p^{\prime}$ when all other players use MAP $p$. The objective of each player is to maximize its own utility. We shall consider the non-cooperative case, which we model as a game (with infinite number of players) and study the existence of symmetric equilibria and their properties. Let $U\left(p^{*}, p^{*}\right)$ 
denote the value of the utility function at the symmetric Nash equilibrum $p^{*}$.

Definition 1: $p^{*} \in\left[\begin{array}{ll}0 & 1\end{array}\right]$ is said to be a symmetric Nash equilibrium if for any node the following holds

$$
U\left(p^{*}, p^{*}\right)=\max _{p \in\left[\begin{array}{ll}
0 & 1
\end{array}\right]} U\left(p, p^{*}\right) .
$$

With some abuse of notation we use the notation $U(p)$ to denote the expected system utility when the same $p$ is used by all players. We consider utilities which attain optimal values for some $p$ and denote it, again with some abuse of notation, as $p_{m}$. It will be clear from the context if $p_{m}$ is an optimizer of the spatial performance metric or the team utility.

In the rest of this section we look for the appropriate utility functions that characterize the performance of the individual player. The next lemma follows from Lemma 3.1 and Corollary 3.2 in [2].

Lemma 1: Consider a Poisson MANET with the assumptions in Corollary 1. Let a typical node transmit with MAP $p^{\prime}$, while all other players transmit with MAP $p$. Then the goodput of the typical node is

$$
g\left(p^{\prime}, p\right)=p^{\prime} \exp \{-p \lambda C\} .
$$

where $C=2 \pi r^{2} T^{2 / \beta} K(\beta)$ is defined earlier.

We note the following:

1) The goodput is monotone increasing in $p^{\prime}$. Hence, if the objective of each mobile is to maximize its goodput, then the only equilibrium is $p=1$ for all mobiles.

2) Under the conditions of the above lemma, the energy consumption of the typical node is proportional to $p^{\prime}$ and does not depend on the MAP $p$ of the other nodes. The ratio between the goodput and the expected energy consumption of the typical node does not depend on $p^{\prime}$. We conclude that any $p$ is an equilibrium when the criterion of each node is that of minimizing the ratio between goodput and energy.

Energy is an important resource in MANETS. Let $W\left(p^{\prime}\right)$ denote the energy consumed by the typical node when it transmits with MAP $p^{\prime}$. In practice, in many applications, the utility related to goodput is not a linear function of the MAP, see for example [3], [5]. Keeping this and the remarks made above in view, we shall be interested in a utility of the form

$$
U\left(p, p^{\prime}\right)=f\left(p, p^{\prime}\right)-\rho W\left(p^{\prime}\right)
$$

for each player, where $f$ gives the performance of interest and $\rho$ is a given constant.

The energy dissipated in each node is directly proportional to its MAP, i.e., we take $W\left(p^{\prime}\right)$ equal to the expected power consumed in each node, which is given as $P p^{\prime}$. For simplicity we assume that each node transmits at unit power. The value of $\rho$ characterizes the per unit energy cost that each node incurs in transmission. We refer to it as price factor.

Assume that the function $f$ is a concave function in $p^{\prime}$ and continuous in $p$; then the arguments in [7][Thm 1] can be used to show the existence of symmetric Nash equilibria. We state this result in the following lemma. The proof is given in Appendix $\mathrm{H}$.

Lemma 2: Assume that the utility function $U\left(p^{\prime}, p\right)$ is concave in $p^{\prime}$ and continuous in $p$. Then an symmetric Nash equilibrium exists.

In the next two sections we consider two utility functions defined in term of goodput and delay as the performance measure with the energy costs. We evaluate the MAP at equilibrium and the corresponding system performance. This system performance is then optimized by searching for the best price factor. The best achievable system performance, at equilibrium is then compared with that evaluated when nodes act as a team.

\section{UTILITY WITH GOODPUT}

Assume that each node of the MANET is interested in maximizing its goodput taking into account the energy cost it incurs. Then by taking $f\left(p^{\prime}, p\right)=g\left(p^{\prime}, p\right)$ in equation (12) we define the utility as

$$
U\left(p^{\prime}, p\right)=g\left(p^{\prime}, p\right)-\rho p^{\prime}=p^{\prime}\{\exp \{-p \lambda C\}-\rho\} .
$$

The objective of a typical node is to choose a MAP that maximizes its utility, i.e.,

$$
p^{\prime} \in \operatorname{argmax}_{0 \leq p^{\prime} \leq 1} U\left(p^{\prime}, p\right) .
$$

This utility function is a linear function in $p^{\prime}$ and convex in $p$. Then an from Lemma (2) a SNE exists. We proceed to calculate the SNE of this game.

When $\rho \geq 1$ the slope of the utility of a typical node is non positive irrespective of the MAP of the other nodes. Then the optimal strategy for each node is to choose $p=0$, which is a dominant strategy and hence an equilibrium. When $\rho<1$ consider the following two cases.

Assume $\rho \geq \exp \{-\lambda C\}$ : In this case the slope of the typical node is always positive. Then the optimal strategy for the typical node is to choose $p=1$ irrespective of the MAP chosen by the other nodes. Thus $p=1$ is a dominant strategy and hence also is a symmetric equilibrium.

Assume $\rho<\exp \{-\lambda C\}$ : If each node other than the typical node chooses a MAP such that

$$
\exp \{-p \lambda C\}=\rho,
$$

then the utility of the typical node in (13) is set to zero and is not affected by its strategy, i.e., the typical node becomes indifferent to its own strategy. Further, if any of the nodes deviates from the MAP that satisfies Equation (14), it will not gain anything given that all other nodes set their MAP value as in (14). Hence the MAP satisfying (14) constitutes a symmetric Nash equilibrium. We summarize the above observations in the following proposition.

Proposition 3: For any given $C, \lambda$, and $\rho>0$

- if $\rho \geq 1$ then $p^{*}=0$ is the SNE;

- if $\exp \{-\lambda C\} \geq \rho$ then $p^{*}=1$ is the SNE;

- if $\exp \{-\lambda C\}<\rho<1$ then

$$
p^{*}=\frac{-\log \rho}{\lambda C}
$$


is a SNE.

The goodput of each node at equilibrium is given by

$$
g\left(p^{*}, p^{*}\right)= \begin{cases}0, & \text { if } \rho \geq 1 \\ \exp \{-\lambda C\}, & \text { if } \rho \leq \exp \{-\lambda C\} \\ \frac{-\rho \log \rho}{\lambda C}, & \text { if } \exp \{-\lambda C\}<\rho<1\end{cases}
$$

With the expression for goodput at equilibrium, we can look for the value of $\rho$ that maximizes it. One can also think that the value of $\rho$ is set by a central agent who is interested in optimizing the network performance at equilibrium. We assume that the objective of the central agent is to maximize the density of success at equilibrium. Then the optimization problem of the central agent is given by

$$
\begin{array}{ll}
\underset{\rho}{\operatorname{maximize}} & \frac{-\lambda \rho \log \rho}{\lambda C} \\
\text { subject to } & \exp \{-\lambda C\}<\rho<1 .
\end{array}
$$

The objective function in (16) is a concave function of $\rho>0$ attaining its maximum at $\rho=1 / e$. If $\lambda C>1$ then $1 / e$ lies within the constraint set and the operator can set $\rho^{*}=1 / e$. Suppose $\lambda C \leq 1$, then the objective function is decreasing in the interval $\exp \{-\lambda C\}<\rho<1$ and the maximum is attained at $\rho^{*}=\exp \{-\lambda C\}$. Then the maximum density of success at equilibrium is

$$
d_{s}\left(p^{*}, p^{*}\right)= \begin{cases}1 /(e C), & \text { if } \lambda C>1 \\ \lambda \exp \{-\lambda C\}, & \text { if } \lambda C \leq 1\end{cases}
$$

Comparing this optimal density of successful transmission at equilibrium with the optimal global density of successful transmission given in (9) we have the following result.

Proposition 4: The global optimal density of successful transmissions can be attained at equilibrium by setting the pricing factor $\rho$ as follows:

$$
\rho^{*}= \begin{cases}1 / e, & \text { if } \lambda C>1 \\ \exp \{-\lambda C\}, & \text { if } \lambda C \leq 1\end{cases}
$$

Also, note that with the pricing factor $\rho^{*}$ in (18), the MAP of each node at equilibrium is the same as that achieving the global optimum as given in (8). Thus by pricing appropriately, one can use the selfish behavior of the players to reach an equilibrium at which the global optimal performance of the MANET is attained.

\section{UTILITY WITH DELAY}

In this section we define the utility of each player in terms of the delay associated in delivering the packet successfully at its receiver and the transmission cost. Let a typical node incurs a cost of $\rho$ units per unit energy dissipated. By taking $f\left(p^{\prime}, p\right)=-t\left(p^{\prime}, p\right)$ in equation (12) we get the utility of the typical node as

$$
U\left(p^{\prime}, p\right)=-\frac{1}{p^{\prime} \exp \{-p \lambda C\}}-p^{\prime} \rho .
$$

where $p^{\prime}$ is the MAP of typical node, and $p$ is the MAP used by all other nodes. Recall that $C=2 \pi r^{2} T^{2 / \beta} K(\beta)$.
The objective of each node is to choose a MAP that maximize its utility function (19). Or equivalently it can be given by the following minimization problem:

$$
\underset{p^{\prime}}{\operatorname{minimize}} \frac{\exp \{p \lambda C\}}{p^{\prime}}+p^{\prime} \rho \quad \text { subject to } 0 \leq p^{\prime} \leq 1 .
$$

For a given value of $p$ the utility function in (19) is a concave function in $p^{\prime}$ and continuous in $p$. Then by Lemma (2), SNE exists. We proceed to calculate the SNE by direct computation.

Differentiating the utility function with respect to $p^{\prime}$, equating to zero and simplifying we get

$$
p^{\prime}=\frac{\exp \{p \lambda \bar{C}\}}{\sqrt{\rho}}
$$

where $\bar{C}=C / 2$. This equation gives the best response of the typical node when every other nodes transmits with probability $p$. If there exists a $p \in\left[\begin{array}{ll}0 & 1\end{array}\right]$ such that $p^{\prime}=p$, then $p$ is the SNE of the game. Hence we look for the conditions when the following condition holds

$$
p=\frac{\exp \{p \lambda \bar{C}\}}{\sqrt{\rho}} .
$$

Lemma 3: Equation in (21) has a solution (not necessarily a probability) if and only if $\lambda \bar{C} e \leq \sqrt{\rho}$.

Proof: Rearranging the terms in equation (21) we write

$$
-p \lambda \bar{C} \exp \{-p \lambda \bar{C}\}=\frac{-\lambda \bar{C}}{\sqrt{\rho}}
$$

The solution to the above equation is given as

$$
p=\frac{-1}{\lambda \bar{C}} W\left(-\frac{\lambda \bar{C}}{\sqrt{\rho}}\right),
$$

where $W(\cdot)$ denotes the Lambert function [10]. On the real line $\mathbb{R}$, the Lambert function exists only for values larger than $-1 / e,[10]$ and takes values on the entire real line. Hence the relation in (22) holds for some value of $p$ if and only if $-\lambda \bar{C} / \sqrt{\rho} \geq-1 / e$.

This lemma gives the condition for the existence of fixed points to the equation (21) for some $p$ which do not necessarily lie in the interval $\left[\begin{array}{ll}0 & 1\end{array}\right]$. We next look at the conditions under which the solution results in a probability. We recall the following properties of Lambert functions:

- The Lambert function is two-valued in the interval $[-1 / e, 0]$. The two branches of the Lambert function denoted as $W_{0}(\cdot)$ and $W_{-1}(\cdot)$ meet at $-1 / e$ and the value of the Lambert function at this point is -1 .

- In the interval $[-1 / e, 0] W_{0}(\cdot)$ is a continuous and increasing function taking value in the interval $[-1,-\infty]$.

- In the interval $[-1 / e, 0] W_{-1}(\cdot)$ is a continuous and decreasing function taking value in the interval $[-1, \infty]$.

Based on the above properties we derive the range of $\rho$ for which an equilibrium probability exists. For some value of $\rho$ their exist two equilibrium points as shown in the following lemma. For notational simplicity we write $\rho_{t}=(e \lambda \bar{C})^{2}$. 


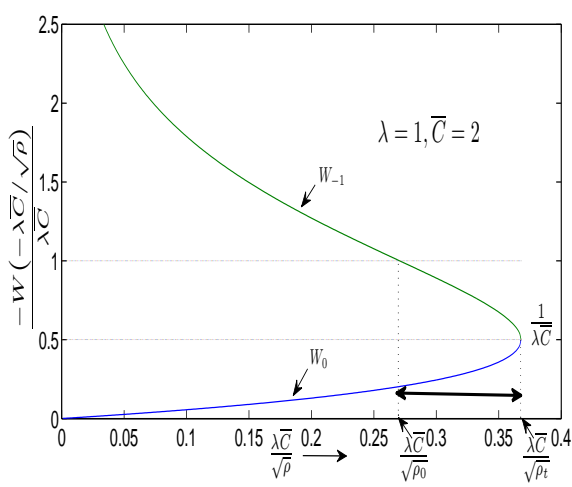

Figure 1. SNE on both branches

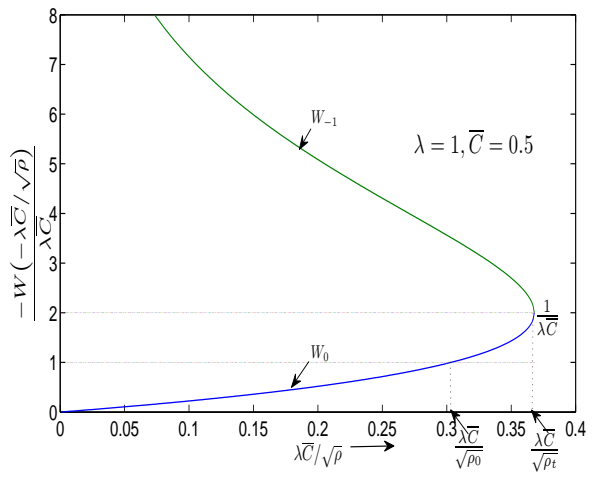

Figure 2. Unique SNE

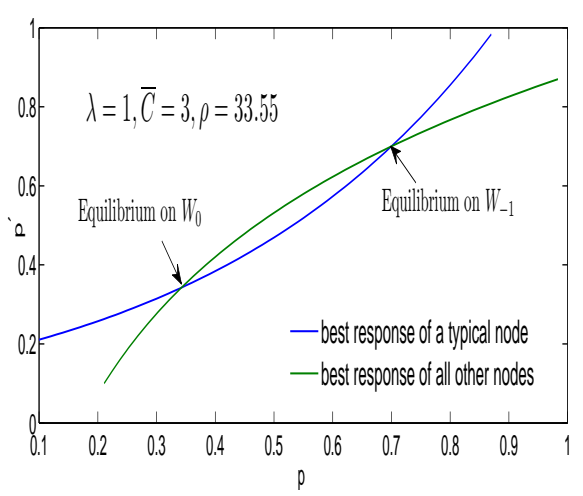

Figure 3. Best Response
Lemma 4: Assume $\lambda \bar{C} \geq 1$, then an equilibrium exists for all $\rho$ satisfying $\rho \geq \rho_{t}$. Further, there exists a $\rho_{-1} \geq \rho_{t}$ such that for all $\rho$ satisfying $\rho_{-1} \geq \rho \geq \rho_{t}$ two equilibrium points are possible. One point lying on the $W_{0}$ and other on the $W_{-1}$ branch.

Proof: See Appendix A.

Note that for the value of $\rho \in\left[\rho_{t}, \rho_{-1}\right]$, the equilibrium MAP computed on the $W_{-1}$ is larger than that computed on the $W_{0}$ branch. If nodes reach the equilibrium that is computed on the $W_{-1}$ branch, then they will be transmitting aggressively at equilibrium. We will see later that this leads to inefficiency (see remark (1)).

Lemma 5: Assume that $\lambda \bar{C}<1$, then there exists $\rho_{0} \geq \rho_{t}$ such that for all $\rho \geq \rho_{0}$ an equilibrium point exists and is unique. This equilibrium point lies on the $W_{0}$ branch.

Proof: See Appendix B.

Lemma 4 and 5 completely characterizes all the possible equilibriums. Figures 1 and 2 illustrate the Lambert functions and its properties used in the proof of Lemma 4 and 5. In Figure 1 the $y$ axis marked with double arrow denotes the region in which two equilibrium points occur.

\section{A. Stability of SNE}

Figure 3 shows the best response of the typical node and that of all nodes against the typical node. This example demonstrate existence of two SNE. The smaller SNE among the two results from the principal branch of the Lambert function in Equation (22), and the larger one from the $W_{-1}$ branch. When two equilibrium points exists we denote the SNE resulting from the $W_{0}$ branch of the Lambert function as $p_{0}^{*}$ and that resulting from the $W_{-1}$ branch as $p_{-1}^{*}$. When we do not need to make this distinction or it is unique we write the symmetric Nash equilibria as $p^{*}$. From Figure 3 we see that at the equilibrium point $p_{0}^{*}$ a slight increase in the MAP $p$ results in a decrease in $p^{\prime}$. This is a stabilizing behavior and we conclude that $p_{0}^{*}$ is stable. In contrast, at equilibrium point $p_{-1}^{*}$ a slight increase in $p$ is seen to cause an increase in the value of $p^{\prime}$. Thus the second equilibrium is unstable.

\section{B. optimal pricing}

Assume that $p^{*}$ is an equilibrium point for a given value of $\rho$ that satisfies the conditions in Lemma 4 or 5 for a given value of $\lambda$ and $\bar{C}$. From Equation (5) the mean delay experienced by the packets of a typical node at equilibrium can be computed as

$$
\begin{aligned}
t & \left(p^{*}, p^{*}\right)=\frac{1}{p^{*} \exp \left\{-p^{*} \lambda C\right\}} \\
& =\frac{1}{p^{*} \exp \left\{-p^{*} \lambda \bar{C}\right\} \exp \left\{-p^{*} \lambda \bar{C}\right\}} \\
& =\frac{\sqrt{\rho}}{\exp \{W(-\lambda \bar{C} / \sqrt{\rho})\}} \\
& =\frac{\sqrt{\rho} W(-\lambda \bar{C} / \sqrt{\rho})}{W(-\lambda \bar{C} / \sqrt{\rho}) \exp \{W(-\lambda \bar{C} / \sqrt{\rho})\}} \\
& =\frac{\sqrt{\rho} W(-\lambda \bar{C} / \sqrt{\rho})}{(-\lambda \bar{C} / \sqrt{\rho})}=-\frac{\rho W(-\lambda \bar{C} / \sqrt{\rho})}{\lambda \bar{C}}
\end{aligned}
$$

In above chain of equalities the relation $C=2 \bar{C}$ is used in Equation (23). Equation (24) follows from equation (21). Equation (26) follows by applying the definition of the Lambert function to the denominator term in Equation (25) and rearranging. From (26) we see that when the SNE is not unique delay experienced by the typical is larger when the SNE results form the $W_{-1}$ branch of the Lambert function.

With the expression for the delay of a typical node at equilibrium, one can look for the value of the price factor that minimizes the delay experienced by each node at equilibrium. We assume that the objective of the central agent who sets the value of $\rho$ is to minimize the average delay per unit area, i.e., spatial delay density, at equilibrium. Then the optimization problem of the central agent is:

$$
\begin{array}{ll}
\underset{\rho}{\operatorname{minimize}} & -\frac{\rho W(-\lambda \bar{C} / \sqrt{\rho})}{\bar{C}} \\
\text { subject to } & \sqrt{\rho} \geq \lambda \bar{C} e \\
\text { and } & -W(-\lambda \bar{C} / \sqrt{\rho}) \leq \lambda \bar{C} .
\end{array}
$$

The first constraint in this optimization problem (27) results from Lemma 3 and the second constraint is required to ensure that the resulting value of $p$ in (21) lies in the interval [ 01$]$.

Let $h(\rho):=-\rho W(-\lambda \bar{C} / \sqrt{\rho})$ denote the objective function in the above optimization problem without the multiplica- 
tive factor. $h(\rho)$ is defined for $\sqrt{\rho} \geq e \lambda \bar{C}$. In the following lemma we state some of its properties.

Lemma 6: On the principal branch $W_{0}, h(\rho)$ is a quasi convex function in $\rho$ and the global minimum is attained at $\rho^{*}=4 e(\lambda \bar{C})^{2}$. On the $W_{-1}$ branch $h(\rho)$ is a monotonically increasing function taking value $(e \lambda \bar{C})^{2}$ at $\rho=(e \lambda \bar{C})^{2}$

Proof: See Appendix C.

Consider the optimization problem in (27) on the $W_{0}$ branch of the Lambert function. The value of $\rho^{*}$ at which $h(\rho)$ achieves minimum satisfies the first condition in (27) as $\rho^{*}=4 e(\lambda \bar{C})^{2} \geq e^{2}(\lambda \bar{C})^{2}$. The value of $p$ in equation (21) at $\rho=\rho^{*}$ is $1 /(2 \lambda \bar{C})$. This implies that whenever $2 \lambda \bar{C}=\lambda C>1$, the resulting value of $p$ lies inside $(01)$, thus satisfying the second condition of the optimization problem. Hence under the assumption $\lambda C>1$ the global minimizer of $h(\rho)$ lies in the constraint set of $(27)$ and the value of the objective function at this point is

$$
-\frac{\rho^{*} W\left(-\lambda \bar{C} / \sqrt{\rho^{*}}\right)}{\lambda \bar{C}}=-\frac{\left(4 e(\lambda \bar{C})^{2}\right)(-1 / 2)}{\lambda \bar{C}}=e \lambda C .
$$

When $\lambda C \leq 1$, any value of $\rho$ such that $\rho<\rho^{*}$ violates the second condition, as $-W(-\lambda \bar{C} / \sqrt{\rho})>1 / 2$. From Lemma 6 the minimum value of $h(\rho)$ is achieved at a $\rho$ that satisfies $-W(-\lambda \bar{C} / \sqrt{\rho})=\lambda \bar{C}$. This implies that under the condition $\lambda C \leq 1$ the value of symmetric equilibrium is $p=1$ and the delay experienced by each node is given by $\exp \{-\lambda C\}$. We summarize these observations in the following proposition.

Proposition 5: The value of the price factor $\rho^{*}$ that minimizes the delay at equilibrium is as follows:

$$
\rho^{*}= \begin{cases}(2 \lambda \bar{C} \sqrt{e})^{2} & \text { if } \lambda C>1 \\ -2 W\left(-\lambda \bar{C} / \sqrt{\rho^{*}}\right)=\lambda C, & \text { if } \lambda C \leq 1\end{cases}
$$

and the corresponding delay at equilibrium is

$$
d_{t}\left(p^{*}, p^{*}\right)= \begin{cases}\lambda^{2} e C & \text { if } \lambda C>1 \\ \lambda \exp \{\lambda C\}, & \text { if } \lambda C \leq 1 .\end{cases}
$$

Comparing Equations (30) and (10) we can conclude the following result:

Proposition 6: The spatial delay density in the game problem at equilibrium equals the global optimal spatial delay density, i.e., $d_{t}\left(p_{m}\right)=d_{t}\left(p^{*}, p^{*}\right)$, if the price factor is chosen as in (29).

Again, by appropriately pricing the nodes the selfish behavior can be used to attain the global optimal performance in the game problem. If the SNE is not unique at the optimal price factor $\rho^{*}$, then one needs to ensure that the nodes reach equilibrium that is computed on the principal branch of the Lambert function to realize the global optimal performance at equilibrium. Indeed, if $\lambda C>1$ and $-W_{-1}(-1 / 2 \sqrt{e}) \leq \lambda \bar{C}$ or equivalently $\rho_{-1} \geq 4 e(\lambda \bar{C})^{2}$, at the optimal price $\rho^{*}$ an equilibrium point exists on the $W_{-1}$ branch of the Lambert function. Thus it is an interesting question to ask how to make the nodes reach a favorable equilibrium at the optimal price factor. However we do not pursue this question in this paper.

Remark 1: If $\lambda \bar{C}>1$ and optimization is restricted to the $W_{-1}$ branch in (27), then by Lemma 6 , the objective function is minimized by choosing $\rho$ satisfying $\sqrt{\rho}=e \lambda \bar{C}$, which results in the equilibrium probability $1 /(\lambda \bar{C})$. The spatial density of delay at this equilibrium point is given by

$$
-\frac{\rho W(-\lambda \bar{C} / \sqrt{\rho})}{\lambda \bar{C}}=\frac{(e \lambda \bar{C})^{2}}{\lambda \bar{C}}=(e / 2) e \lambda C .
$$

Comparing this value with (10), we see that spatial density of delay increased by a factor of $e / 2$ by the selfish behavior of the nodes.

\section{PRICE OF ANARCHY}

In this section we study the degradation in the network performance due to a selfish behavior of the nodes. Price of Anarchy (PoA) compares the social utility at the worst equilibrium with the optimal social utility [21]. For our Poisson bipolar MANET with infinitely many players, we define the price of anarchy as the ratio of the optimal spatial average performance that can be achieved, to the spatial average performance at the worst SNE. Recall that we denoted the system utility by $U(p)$ when we considered the team problem, with each node using the same MAP $p$. For the game problem we denoted the utility of a typical node by $U\left(p^{\prime}, p\right)$. In the game problem the spatial average performance at equilibrium is evaluated by multiplying the utility of the typical and the intensity of the Poisson point process . Then PoA is given by

$$
P o A=\frac{\max _{p \in[01]} U(p)}{\min _{p^{*} \in S} \lambda U\left(p^{*}, p^{*}\right)},
$$

where $S \subset\left[\begin{array}{ll}0 & 1\end{array}\right]$ denotes the set of symmetric Nash equilibria.

We study PoA as a function of $\rho$ for a given value of $\lambda$ and $C$. The utilities studied in Section $\mathrm{V}$ and VI are considered below.

\section{A. Goodput}

In this subsection we consider the utility defined in Section $\mathrm{V}$. Let us begin by considering the team utility. When all the nodes use the MAP $p$, then the from equation (13) the team utility is given by

$$
U(p)=\lambda p \exp \{-p \lambda C\}-\lambda p \rho .
$$

If $\rho>1$ the maximum value of the utility is zero and the maximum is attained at $p_{m}=0$. Let $p_{m}:=p_{m}(\rho)$ denote the MAP that maximizes the team utility in (32). The following lemma gives its value.

Lemma 7: The MAP value that maximizes the the team utility (32) is given by

$$
p_{m}=\frac{1-W(\rho e)}{\lambda C}
$$

for all $\rho \geq 0$ if $\lambda C \geq 1$, and if $\lambda C<1$ it is the maximizer for $\rho$ such that $W(\rho e) \geq 1-\lambda C$. Further the maximum team utility is given by

$$
U\left(p_{m}\right)=\frac{\rho(1-W(\rho e))^{2}}{C W(\rho e)} .
$$

Proof: See Appendix I. 


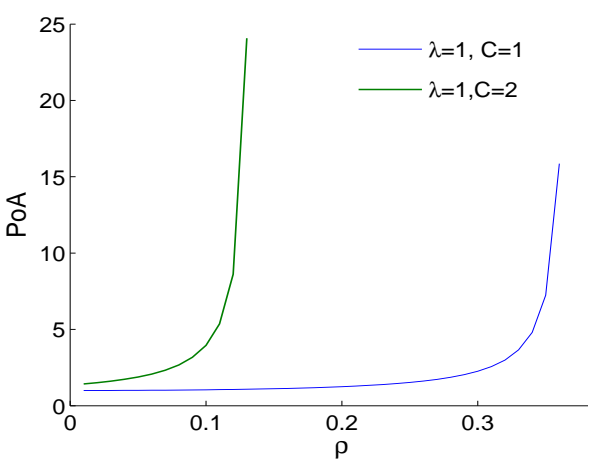

Figure 4. PoA for Goodput

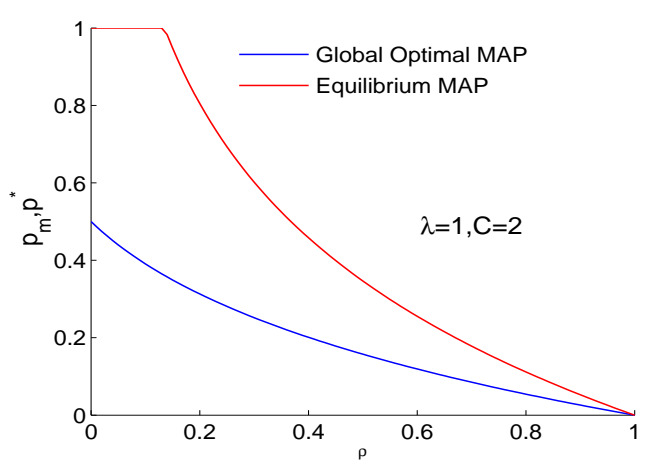

Figure 5. Equilibrium and Optimal MAP

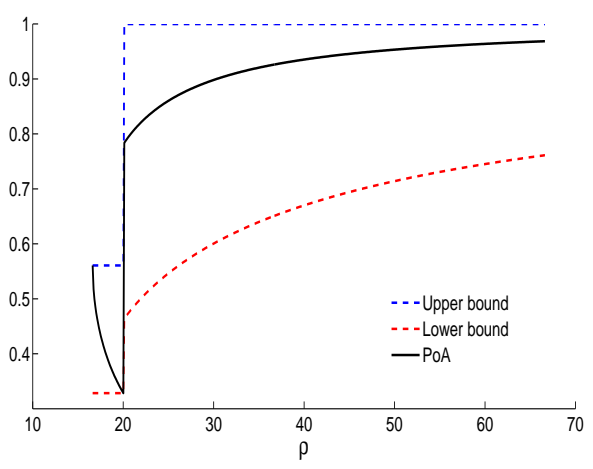

Figure 6. PoA for delay, $\mathrm{C}=3, \lambda=1$
By using definition of the Lambert function one can verify that $U\left(p_{m}\right)$ is a decreasing function in $\rho$. Indeed, differentiating $U\left(p_{m}\right)$ with respect to $\rho$ we have

$$
\begin{aligned}
\frac{\partial U\left(p_{m}\right)}{\partial \rho} & =\frac{(1-W(\rho e))^{2}}{C W(\rho e)} \\
& -\rho e \frac{(1-W(\rho e))}{C W(\rho e)} \frac{\left(1+W(\rho e) W^{\prime}(\rho e)\right.}{C W(\rho e)} \\
& =\frac{(1-W(\rho e))^{2}}{C W(\rho e)}-\frac{(1-W(\rho e))}{C W(\rho e)}
\end{aligned}
$$

In equation (55) $W^{\prime}$ denotes the derivative of Lambert function. Equation (56) follows by applying formula for the derivative of Lambert function. The last equation is negative valued for all $\rho \in[0,1]$. Thus the optimal utility is a decreasing function in $\rho$.

Let us look at the utility of the typical node at equilibrium. From Proposition 3 we have

$$
U\left(p^{*}, p^{*}\right)= \begin{cases}\exp \{-\lambda C\}-\rho, & \text { if } \rho \leq \exp \{-\lambda C\} \\ 0, & \text { if } \rho \geq \exp \{-\lambda C\}\end{cases}
$$

The utility at equilibrium is also a decreasing function in $\rho$ for all $\rho \leq \exp \{-\lambda C\}$. With the expression for utility at equilibrium and global optimum we have the following result for PoA

Proposition 7: The value of PoA is as follows

$$
\operatorname{Po} A(\rho)= \begin{cases}\frac{\rho(1-W(\rho e))^{2}}{\lambda C W(\rho e)\{\exp \{-\lambda C\}-\rho\}}, & \text { if } \rho<\exp \{-\lambda C\} \\ \infty, & \text { if } \rho \geq \exp \{-\lambda C\}\end{cases}
$$

PoA as a function of $\rho$ is shown in figure 4. From this figure we see that as $\rho$ increases the value of PoA grows unboundedly. Thus the PoA is optimal when the pricing factor is set to zero. If $\lambda C \geq 1$ then the PoA is infinite by definition at $\rho=1 / e$. However we noted in the Section V that optimal performance of the spatial density of success is achieved at equilibrium with the same price factor. If $\lambda C<1$ then the PoA is infinite at $\rho=\exp \{-\lambda C\}$. But again we noted in the Section $\mathrm{V}$ that at this price factor optimal performance of the spatial density of success is achieved at equilibrium.

In figure 5 the equilibrium MAP and global optimal MAP are shown. For all values of $\rho$ equilibrium MAP is larger than global optimal MAP. Hence the nodes transmit more aggressively at equilibrium. But we note from the figure 5 that the gap between the global optimal MAP and equilibrium MAP reduces with pricing.

\section{B. Delay}

Consider the utility function in Equation (19). The team utility for this game when each node transmits with MAP $p$ is

$$
U(p)=\frac{-\lambda}{p \exp \{-p \lambda C\}}-\lambda \rho p .
$$

It is easy to verify that the above utility function is concave in $p$. Assume that $\lambda C>1$. Then the unique MAP, denoted as $p_{m}:=p_{m}(\rho)$ that maximizes the social utility satisfies

$$
\exp \left\{p_{m} \lambda C\right\}\left(1-p_{m} \lambda C\right)=\rho p^{2} .
$$

We obtain this by differentiating Equation (37) and setting to zero. Note that any $p_{m}$ that satisfies Equation (38) also satisfies $p_{m} \lambda C \leq 1$, hence $p_{m} \in\left[\begin{array}{ll}0 & 1\end{array}\right]$. Also, it can be easily verified that $p_{m}$ is decreasing in $\rho$.

Utility at equilibrium can be obtained by using the equilibrium MAP in (22) and Equation (26), which is given by

$$
U\left(p^{*}, p^{*}\right)=(-2 \lambda \rho / \lambda \bar{C}) W\left(\frac{-\lambda \bar{C}}{\sqrt{\rho}}\right) .
$$

When $\lambda C>1$, from Lemma 4, two symmetric Nash equilibria are possible for the price factor $\rho \leq \rho_{-1}$. Hence the above utility function can take two values, one corresponding to each equilibrium. Recall that we denoted by $p_{0}^{*}$ the SNE computed on the principal branch, and by $p_{-1}^{*}$ that computed on the other branch of the Lambert function. Recall that $p_{0}^{*} \leq p_{-1}^{*}$. The following proposition gives a bound for the price of anarchy

Proposition 8: For Poisson bipolar MANETS with utility in (19),

$$
\begin{array}{r}
\frac{p_{m}\left(\rho_{-1}\right) \lambda\left(2-p_{m}\left(\rho_{-1}\right) \lambda C\right)}{2\left(1-p_{m}\left(\rho_{-1}\right) \lambda C\right)} \leq \operatorname{PoA}(\rho) \\
\leq \frac{p_{m}\left(\rho_{t}\right) \lambda \bar{C}\left(2-p_{m}\left(\rho_{t}\right) \lambda C\right)}{2\left(1-p_{m}\left(\rho_{t}\right) \lambda C\right)}
\end{array}
$$

for $\rho \in\left[\rho_{t} \rho_{-1}\right]$, where $\sqrt{\rho_{0}}=e \lambda \bar{C}$. And

$$
\frac{p_{m}}{p_{0}^{*}} \leq \operatorname{Po} A(\rho) \leq 1 \text { for } \rho \geq \rho_{-1} \text {. }
$$


Proof: See Appendix D.

The price of anarchy as a function of $\rho$ and the bounds obtained in Proposition 8 are shown in Figure 6. The jump in the figure at $\rho=\rho_{-1}$ is due to two possible symmetric equilibria for $\rho \leq \rho_{-1}$ and unique symmetric equilibrium for $\rho>\rho_{-1}$. In the interval $\left[\rho_{t}, \rho_{-1}\right]$ PoA is decreasing in $\rho$. This results from the bad Nash equilibrium that occurs on the $W_{-1}$ branch of the Lambert function which increases in $\rho$. If the central agent can't set a price factor higher than $\rho_{-1}$ then from the PoA point of view it is desirable to set the lowest possible price factor, i.e., $\rho=\rho_{t}$. For a price factor larger than $\rho_{-1}$ there is unique SNE, which is smaller than the equilibrium that occurs on the $W_{-1}$ branch and decreases ${ }^{1}$ with $\rho$. Thus setting high a price factor leads to improved PoA.

If $\rho_{-1}<4 e(\lambda \bar{C})^{2}$, from Proposition 6 we can see that by setting $\rho=4 e(\lambda \bar{C})^{2}$ one can obtain better performance at equilibrium and also good price of anarchy. If $\rho_{-1}>4 e(\lambda \bar{C})^{2}$ then by setting $\rho=4 e(\lambda \bar{C})^{2}$ one obtains global optimal performance at equilibrium provided the nodes settle at an equilibrium that lies on the principal branch of the Lambert function, otherwise this price factor leads to a poor price of anarchy.

\section{CONCLUSIONS}

Geometric considerations play a very central role in wireless communications, since the attenuation of wireless channels strongly depend on the distance between transmitter and receiver. Models that take into account the exact location of mobiles are often too complex to analyze or to optimize. Our objective in this paper is to model competition between mobiles as a game in which the locations of players is given by a Poisson point process.

The Poisson assumption is valid when

- the number of mobiles in disjoint sets are independent;

- the number of mobiles in any given set follows a Poisson distribution. This class of point processes maximizes entropy. It is often used for modeling the location of users in e.g. mobile ad hoc networks.

More structured point processes can also be contemplated, for instance exhibiting attraction (hot spots) or repulsion (more elaborate medium access control than Aloha like e.g. CSMA). We leave the analysis of medium access games under such point processes for future research.

The competition we considered in the paper was between individual mobiles each taking its own selfish decisions. We saw that the equilibrium of the game results in a more aggressive access (larger access probabilistically). We studied further pricing, and identified pricing parameters that induce an equilibrium achieving the social optimal performance. On the other hand we showed that the utility at equilibrium can be zero).

We plan in the future to study other games within this framework: ones with finitely many operators each taking decisions for all its subscribers. In addition we shall study jamming games.

\footnotetext{
${ }^{1}$ Principle branch of Lambert function is decreasing function of $\rho$
}

\section{REFERENCES}

[1] D. Bertsekas and R. Gallager, Data Networks, Prentice Hall, Englewood Cliffs, New Jersey, 1987.

[2] Baccelli, B. Blaszczyszyn and P. Múhlethaler, "An ALOHA protocol for multihop mobile wireless networks," IEEE Transactions on Information Theory, Vol 52, No. 2, pp. 421 - 436, Feb. 2006.

[3] International Telecommunication Union, "The E-model, a computational model for use in transmission planning," ITU-Recommendation G. 107, July 2000 .

[4] A. A. Kherani, R. El-Azouzi and E. Altman,"Stability-throughput tradeoff and routing in multi-hop wireless ad-hoc networks," proceedings of IFIP Networking, Coimbra, Portugal, May 15-19, 2006. Received the best paper Award.

[5] J. Matta, C. Pepin, K. Lashhkari and R. Jain, " source and channel rate adaptation algorithm for AMR in VoIP using the e-midel," Proc. of the 13th Internationial Workshop on Network and Operating System Support for Digital Audio and Video, pp. 92-99, Montnerey, CA, USA, June 2003.

[6] J. Mo and J. Walrand. "Fair end-to-end window-based congestion control," In SPIE '98, International Symposium on Voice, Video and Data Communications, 1998.

[7] J. Rosen, "Existence and uniqueness of equilibrium points for concave n-person games," Econometrica, vol. 33, pp. 520-534, 1965.

[8] F. Baccelli and B. Blaszczyszyn, "Stochastic Geometry and Wireless Networks Volume 1: THEORY," Foundations and Trends in Networking: Vol. 3: No 3-4, 2009, pp 249-449. http://dx.doi.org/10.1561/1300000006

[9] F. Baccelli and B. Blaszczyszyn, "Stochastic Geometry and Wireless Networks Volume 2: APPLICATIONS," Foundations and Trends in Networking: Vol. 4: No 1-2, 2009, pp 1-312. http://dx.doi.org/10.1561/1300000026

[10] R. M. Corless, G. H. Gonnet, D. E. G. Hare, D. J. Jeffrey, D. E. Knuth, "On the Lambert W function," Advances in Computational Mathematics (Berlin, New York: Springer-Verlag) vol. 5, 1996, pp 329-359

[11] S. Boyd, L. Vandenberghe, "Convex Optimization," Cambridge University Press 2004.

[12] F. Baccelli, B. Blaszczyszyn, P. Múhlethaler, "Stochastic Analysis of Spatial and Opportunistic Aloha," IEEE journal on selected areas in communications, vol. 27, no. 7, sep 2009, pp 1105-1119

[13] G. Hardin, "The Tragedy of the Commons," Science 13 December 1968: Vol. 162 no. 3859 pp. 1243-1248

[14] L. Kleinrock, "Queueing Systems. Volume 1: Theory," WileyInterscience; 1 edition

[15] H. Inaltekin, S. B. Wicker, "The Analysis of nash Equilibria of the OneShot Random-Access Game for Woreless Networks and the Behavior of Selfish Nodes," IEEE/ACM Transactions on Networking, Vol. 16. 5, oct 2008

[16] A.B. Mackenzie, S.B. Wicker, "Stability of multipacket slotted Aloha with selfish users adn perfect informations," in Proc. IEEE INFOCOM, 2003, pp. 1583-1790

[17] Jeffrey G. Andrews, Francois Baccelli, R. K. Ganti, "A New Tractable Model for Cellular Coverage," 48th Allerton conference, Sep. 2010

[18] E. Altman, R. El Azouzi, T. Jimenez, "Slotted Aloha as a stochastic game with partial information," in WiOpt'03, Mar 2003.

[19] Eitan Altman, Anurag Kumar, Chandramani Kishore Singh, Rajesh Sundaresan, "Spatial SINR Games Combining Base Station Placement and Mobile Association.” In Proceedings of INFOCOM'2009. pp.1629 1637

[20] C.U. Saraydar, N.B. Mandayam, D.J. Goodman, "Pricing and power control in multiple wireless data networks" IEEE JSAC, vol.19, no. 10, pp. 1883-1892, Oct, 2001

[21] E. Koutsoupias, C. Papadimitriou, "Worst-case Equilibria," STAC'99, LNCS 1563m pp. 404-413 1999.

\section{APPENDIX A \\ ProOF OF LEMMA 4}

First consider the $W_{0}$ branch of the Lambert function. As $\rho$ takes value in the interval $\left[(\lambda \bar{C} e)^{2} \infty\right], W_{0}\left(\frac{-\lambda \bar{C}}{\sqrt{\bar{\rho}}}\right)$ increases continuously from -1 to 0 . Thus (21) has a solution in the interval $\left[\begin{array}{ll}0 & 1\end{array}\right]$ if $\lambda \bar{C} \geq 1$. This implies that equilibrium point exists on the $W_{0}$ branch for all $\rho$, satisfying $\sqrt{\rho} \geq \lambda \bar{C} e$. 
The $W_{-1}\left(\frac{-\lambda \bar{C}}{\sqrt{\rho}}\right)$ branch decreases continuously from -1 to $-\infty$ as $\rho$ takes value in the interval $\left[(\lambda \bar{C} e)^{2} \infty\right]$. This implies that there exists a $\sqrt{\rho}_{-1} \geq \lambda \bar{C} e$ such that $\left.W_{-1}\left(\frac{-\lambda \bar{C}}{\sqrt{\rho}-1}\right)\right)=$ $-\lambda \bar{C}$, and for all $\rho$ such that $\sqrt{\rho}_{-1} \geq \sqrt{\rho} \geq \bar{C} e$ satisfies $-W_{-1}\left(\frac{-\lambda \bar{C}}{\sqrt{\rho}}\right) \leq \lambda \bar{C}$, resulting in a $p \in\left[\begin{array}{ll}0 & 1\end{array}\right]$ that is a solution of (21). Hence there exists an equilibrium point on the $W_{-1}$ branch for all $\rho$ satisfying $\sqrt{\rho}_{-1} \geq \sqrt{\rho}$. This concludes the proof.

\section{APPENDIX B}

\section{PROOF OF LEMMA 5}

As in Lemma 4 we can argue that on the $W_{0}$ branch, there exists $\rho_{0} \geq \lambda \bar{C} e$ such that $W_{0}\left(-\frac{\lambda \bar{C}}{\sqrt{\rho_{0}}}\right)=-\lambda \bar{C}$ and for all $\rho$ such that $\rho \geq \rho_{-1} \geq \lambda \bar{C} e$ satisfies $-W_{0}\left(\frac{-\lambda \bar{C}}{\sqrt{\rho}}\right) \leq \lambda \bar{C}$ as $-W_{0}$ is a decreasing function $\rho$.

\section{APPENDIX C}

\section{PROOF OF LEMMA 6}

Differentiating $h(\rho)$ with respect to $\rho$

$$
\begin{aligned}
& \frac{\mathrm{d}}{\mathrm{d} \rho} h(\rho) \\
& \quad=-W(-\lambda \bar{C} / \sqrt{\rho})-W^{\prime}(-\lambda \bar{C} / \sqrt{\rho})(\lambda \bar{C} / 2 \sqrt{\rho}) \\
& \quad=-W(-\lambda \bar{C} / \sqrt{\rho})\left(1-\frac{1}{2(1+W(-\lambda \bar{C} / \sqrt{\rho}))}\right)
\end{aligned}
$$

In Equation (43) $W^{\prime}(\cdot)$ denotes the derivative of the Lambert function which is given as [10][eqn. 3.2]

$$
W^{\prime}(x)=\frac{W(x)}{x(1+W(x))} \text { for } x \neq 0, x \neq-1 / e .
$$

Equation (44) is obtained by substituting the derivative in (45), evaluated at $x=-\lambda \bar{C} / \sqrt{\rho}$, in Equation (43). Recall that on the principal branch of the Lambert function $W(-\lambda \bar{C} / \sqrt{\rho})$ is a negative valued increasing function in $\rho$. Then the term within parenthesis in (44) is a increasing function of $\rho$ passing through the origin at $\rho^{*}$ that satisfies $W\left(-\lambda \bar{C} / \sqrt{\rho^{*}}\right)=-1 / 2$. Thus $h(\rho)$ is decreasing for $\rho \leq \rho^{*}$ and increasing for $\rho \geq \rho^{*}$. From [11][sec. 3.4.2] we conclude that $h(\rho)$ is a quasi convex function in $\rho$.

Further by the definition of the Lambert function

$$
\begin{aligned}
-\lambda \bar{C} / \sqrt{\rho^{*}} & =W\left(-\lambda \bar{C} / \sqrt{\rho^{*}}\right) \exp \left\{W\left(-\lambda \bar{C} / \sqrt{\rho^{*}}\right)\right\} \\
& =-\frac{1}{2} \exp \{-1 / 2\} .
\end{aligned}
$$

Rearranging Equation (46), we get $\rho^{*}=4 e(\lambda \bar{C})^{2}$. The other part of the Lemma follows by noting that $-W(-\lambda \bar{C} / \sqrt{\rho})$ is an increasing function in $\rho$ on the $W_{-1}$ branch.

\section{APPENDIX D}

\section{PROOF OF PROPOSITION 8}

From Equation (39) and (37) we have

$$
\begin{aligned}
\operatorname{PoA}(\rho) & =\frac{\exp \left\{p_{m} \lambda C\right\} / p_{m}+\rho p_{m}}{(-2 \rho / \lambda \bar{C}) W\left(-\frac{\lambda \bar{C}}{\sqrt{\rho}}\right)} \\
& =\frac{p_{m} /\left(1-p_{m} \lambda C\right)+p_{m}}{(-2 / \lambda \bar{C}) W\left(-\frac{\lambda \bar{C}}{\sqrt{\rho}}\right)} \\
& =\frac{p_{m}\left(2-p_{m} \lambda C\right)}{(-2 / \lambda \bar{C}) W\left(-\frac{\lambda \bar{C}}{\sqrt{\rho}}\right)\left(1-p_{m} \lambda C\right)} \\
& \geq \frac{p_{m}}{(-1 / \lambda \bar{C}) W\left(-\frac{\lambda \bar{C}}{\sqrt{\rho}}\right)} .
\end{aligned}
$$

We arrive at equality (48) by dividing both numerator and denominator in (47) by $\rho$, and applying the relation in (38). Equality (49) is obtained by simple rearrangement of terms in the previous step.

To derive the bounds in (40), we consider the equilibrium computed on the $W_{-1}$ branch of the Lambert function as it leads to the worst case equilibrium. This equilibrium is an increasing function in $\rho$ in the interval $\rho_{0} \leq \rho \leq \rho_{-1}$ as discussed in the proof of Lemma (4). Also, recall that the value of $p_{m}$ is decreasing in $\rho$. Thus the numerator in (48) is decreasing in $\rho$. Which implies that the ratio in (49) is also decreasing in $\rho$. The upper bound in (40) now follows by noting that $-W\left(\rho_{0}\right)=1$. To obtain the lower bound we use the relation $-W\left(-\lambda \bar{C} / \sqrt{\rho_{-1}}\right)=\lambda \bar{C}$ in (50).

For values of $\rho$ larger than $\rho_{-1}$ the SNE is unique, resulting from the principal branch of the Lambert function. The upper bound in (42) follows directly by the definition of PoA, and the lower bound follows from the Inequality (50). Note that the lower bound is a function of $\rho$.

\section{APPENDIX E \\ APPENDIX F \\ PROOF OF PROPOSITION \\ APPENDIX G \\ PROOF OF LEMMA \\ APPENDiX H \\ PROOF OF LEMMA 2}

Consider a point to set map $\delta:[0,1] \rightarrow[0,1]$ defined by

$$
\delta(p)=\left\{p^{\prime} \mid U\left(p^{\prime}, p\right)=\max _{q \in[0,1]} U(q, p)\right\} .
$$

This defines the set of best responses of the typical node when all the other nodes use the MAP $p$. It follows from the continuity of $U\left(p^{\prime}, p\right)$ in $p$, and concavity in $U\left(p^{\prime}, p\right)$ for a fixed $p$ that $\Gamma$ is an upper continuous mapping that maps each point of the set $[0,1]$ into a subset of $[0,1]$. By the Kakutani fixed point theorem, there exists a point $p^{*} \in[0,1]$ such that

$$
U\left(p^{*}, p^{*}\right)=\max _{p^{\prime} \in[0,1]} U\left(p^{\prime}, p^{*}\right) .
$$

Then $p *$ is the symmetric Nash equilibrium by Definition 1 . 


\section{APPENDIX I}

\section{PROOF OF LEMMA 7}

Assume $\rho \leq 1$, then $p_{m}>0$ and satisfies the equation

$$
\exp \left\{-p_{m} \lambda C\right\}\left(1-p_{m} \lambda C\right)=\rho \text {. }
$$

By rearranging, $p_{m}$ can be expressed

$$
p_{m}=\frac{1-W(\rho e)}{\lambda C} .
$$

Recall that $W(\cdot)$ is a monotonically increasing function taking values $W(0)=0$ and $W(e)=1$. If $\lambda C \geq 1$ the $p_{m}$ lies in the interval $[0,1]$ for all $\rho \in[0,1]$. If $\lambda C<1$, then $p_{m}$ lies in the interval $[0,1]$ for all $\rho$ such that $W(\rho e) \geq 1-C \lambda$. Thus whenever $\lambda C<1$ we assume that $\rho$ satisfies $W(\rho e) \geq 1-C \lambda$. Then we can compute the optimal value of the utility function as

$$
\begin{aligned}
p_{m} & \exp \left\{p_{m} \lambda C\right\} \\
= & \frac{1-W(\rho e)}{\lambda C} \exp \{-1+W(\rho e)\} \\
& =\frac{1}{e \lambda C}\{\exp \{W(\rho e)\}-\rho e\} \\
& =\frac{1}{e \lambda C}\left\{\frac{\rho e}{W(\rho e)}-\rho e\right\} \\
& =\frac{\rho}{\lambda C}\left\{\frac{1}{W(\rho e)}-1\right\}
\end{aligned}
$$

where Equation (51) is obtained by substituting the value of MAP maximizes the team utility. Equation ()52) and (53) follows by application of the definition of the Lambert function. The maximum utility for the team case can be now computed as a function of $\rho$

$$
U\left(p_{m}\right)=\frac{\rho(1-W(\rho e))^{2}}{C W(\rho e)} .
$$

Differentiating this equation with respect to $\rho$, and simplifying we get

$$
\begin{aligned}
\frac{\partial U\left(p_{m}\right)}{\partial \rho} & =\frac{(1-W(\rho e))^{2}}{C W(\rho e)} \\
& -\rho e \frac{(1-W(\rho e))}{C W(\rho e)} \frac{\left(1+W(\rho e) W^{\prime}(\rho e)\right.}{C W(\rho e)}(55) \\
& =\frac{(1-W(\rho e))^{2}}{C W(\rho e)}-\frac{(1-W(\rho e))}{C W(\rho e)}
\end{aligned}
$$

In equation (55) $W^{\prime}$ denotes the derivative of Lambert function. Equation (56) follows by applying the definition of Lambert function in (45). The last equation is negative values for all $\rho \in[0,1]$ we thus conclude the optimal utility is a decreasing function in $\rho$. 